
\title{
25 Research Square \\ Medical Students Describe Their Wellness And How To Preserve It
}

Krishanu Chatterjee ( $\nabla$ kchatt5@uic.edu )

Mayo Clinic Alix School of Medicine, Mayo Clinic

Victoria S. Edmonds

Mayo Clinic Alix School of Medicine, Mayo Clinic

Marlene E. Girardo

Mayo Clinic

Kristin S Vickers

Mayo Clinic

Julie C Hathaway

Mayo Clinic College of Medicine and Science

Cynthia M. Stonnington

Mayo Clinic

\section{Research Article}

Keywords: Education environment, qualitative analysis, Undergraduate medical education, student wellness, wellness curriculum

Posted Date: February 28th, 2022

DOI: https://doi.org/10.21203/rs.3.rs-1330888/v1

License: (c) (1) This work is licensed under a Creative Commons Attribution 4.0 International License.

Read Full License 


\section{Abstract}

Background: Despite widespread efforts to create wellness programming in medical schools, there is a paucity of literature examining students' perception of wellness and perceptions of these programs. With the inaugural class at the Arizona campus of Mayo Clinic Alix School of Medicine (MCASOM-AZ), an opportunity arose to establish an empirically evaluated wellness curriculum that most inclusively and effectively enables medical students to flourish for years to come. The initial wellness offerings included mental health, academic success, and disability services, curriculum-embedded seminars, wellness committee driven programming, and student-proposed wellness activities. We aimed to improve the relevance and impact of medical school wellness curricula by soliciting in-depth and longitudinal perspectives of medical students themselves. As MCASOM-AZ opened in 2017, the student body at the time of study consisted of first- and second-year medical students.

Methods: Employing a mixed methods analysis of qualitative and longitudinal quantitative data, first- and second-year students at a MCASOM-AZ were invited to respond to an anonymous, online yearlong survey (baseline, six months and 12 months) during the 2018-2019 academic year and participate in a structured, in-depth and in-person, peer-to-peer interview about their conceptions of wellness and the MCASOM-AZ wellness curriculum and resources. Qualitative data was coded for themes using thematic analysis strategies by independent raters.

Results: Nearly half of eligible students completed the baseline survey, $1 / 3$ completed all 3 time-points, and $1 / 5$ participated in an in-depth interview. Participant age, gender, and year of school were representative of the larger student body. Although individual conceptions varied, Wellness was consistently highly valued. Family, Academic Performance, and Friends emerged as most important to well-being across time-points. Academic work arose as the largest barrier to wellness. Analysis of qualitative data revealed five themes. Despite individual differences in approaches to wellness, wellbeing was interrelated to the learning environment; mandatory wellness efforts that didn't address the medical culture met with skepticism.

Conclusions: Interview responses provided understanding and context by which to interpret questionnaire responses. Academics was critical to students' identity and wellness, while also the largest barrier. Suggested curricular improvements include restructuring academic work, seamlessly integrating wellness within coursework, and offering optional individualized approaches.

\section{Background}

In recent years, student wellness during medical school has garnered much needed attention. The increased awareness of physician burnout and depression,[1,2] which has been shown to begin in medical school,[3] motivated individual medical schools as well as the governing bodies of the AMA and AAMC to launch programs to assess levels of distress in medical students $[4,5]$ and to identify the specific stressors responsible.[6-8] The pressure to score highly on examinations, stigma around help- 
seeking, and a surrounding culture that prioritizes performance over wellness have been a few of the drivers implicated. $[9,10]$ These findings have cumulatively led to curricular changes in medical schools throughout the country such as pass/fail grading and integration of wellness programs,[11] as well as the recognition that maintaining wellness ought to be a core physician competency.[12] Despite the paucity in empirically evaluated interventions,[13] the promise of fostering students' resilience and professional development via expanded wellness programs at medical school is compelling.[11]

The input of medical students themselves has been recognized as crucial to the development and employment of these changes.[14,15] Collection of qualitative data from students is one way to gather such insights. In previous studies, medical students were recruited to identify their most salient stressors as well as the self-care behaviors they use to cope with these stressors[16, 17] in order to build the framework for curricular change.[18] Similarly, in other medical school populations, written responses were gathered to open-ended questions regarding the stressors and barriers to wellness encountered in medical school.[18-20] Another compelling study gathered verbal responses to a wide-ranging set of questions about their entire medical school experience through "life-story interviews" with medical students who had already undergone the NRMP match.[21] None of these studies, however, sought a mixed methodology, where the details identified through qualitative means could be correlated with longitudinal quantitative survey measures among the same cohort. Such mixed methodology adds context and nuance to the quantitative data and thus may help to understand why some well-intentioned programs or curricular changes succeed or fail. Furthermore, a thorough exploration of how students personally conceptualize the wellness that is at stake in this topic appears lacking in the present literature. Since the topic of wellness is inherently subjective and driven by unique personal values, there is a challenge to align curricular changes with these diversely-held attitudes and approaches towards personal wellness.

With the inaugural class at the Arizona campus of Mayo Clinic Alix School of Medicine (MCASOM-AZ), an opportunity arose to refine a wellness curriculum that is optimally valued by its students. Our long-term goal was to establish an empirically evaluated wellness curriculum that most inclusively and effectively enables students to flourish during medical school for years to come. To this end, first and second year student perspectives on wellness, including their individual definitions of wellness, strategies for maintaining wellness, barriers to wellness, and attitudes about the existing wellness curriculum, were gathered qualitatively in the form of sit-down interviews conducted by their peers. This was in addition to surveys that assessed repeated categorical and validated measures of student wellness at three timepoints over one year. We recently reported details of the initial wellness curriculum and results of the categorical survey data, which showed significant increase in wellbeing and decrease in perceived stress over the course of the year, with no difference between students who participated in the wellness curriculum and those who didn't.[22] Furthermore, students perceived unscheduled time as being most impactful to their wellness and student-initiated activities as second-most impactful.[22]

This study's primary aims were to uncover key themes in how medical students describe and maintain their wellness, their solutions to perceived barriers, why they preferred unstructured time or student-led 
curriculum to faculty-led wellness curriculum, and to further understand the reasons for our findings in the quantitative and longitudinal data reflecting changing preferences in activities that helped to maintain wellness. We employed a mixed methods approach of gathering verbal, qualitative responses to relevant prompts anchored on individual conceptualizations of wellness, and cross-analyzing this data with responses to related questions over time among the same student cohort. Importantly, the administration of the interviews and surveys were conducted by peers, allowing a level of trust and collegiality that is crucial for gathering honest responses. The insights from this study helped refine our wellness programing, and we expect our approach and findings to inform other medical schools with their curricula and wellness programming.

\section{Methods}

\section{Participant Recruitment}

All 100 MCASOM-AZ students were asked to participate in both the quantitative (survey-based) and qualitative (interview-based) components of this study, and of these 49 agreed to participate. As MCASOM-AZ opened in 2017, all recruited participants were year 1 or 2 students. Participation was elective and all responses were anonymous. The Mayo Clinic Institutional Review Board and the Mayo Clinic College of Medicine and Science Education Research Committee approved this study (protocol number 18-005605). We informed participants that participation was voluntary and that their current and future employment, education, and medical care at Mayo Clinic would not be affected by whether or not they participated. Participants received a $\$ 20$ incentive for completing all three surveys, or for completing the in-person interview.

\section{Quantitative Data Collection}

We surveyed participants electronically at three points during the 2018-2019 academic year. We distributed a "baseline" survey in July 2018, a "midterm" 6 month follow-up survey in January 2019, and a "final" 1 year follow-up survey in June 2019. We explained to participants via email that the purpose of the survey was to ask students about their perceived wellness and personal beliefs and attitudes towards wellness in order to develop a more effective and inclusive wellness curriculum.

Participants generated an anonymous unique identifying code that was used to link their data over the course of the study. Questionnaire 1 was distributed at all three time points, collected two demographics of gender and age group, and gathered responses for perceived factors influencing wellness and success as a medical student (available as Supplemental Digital Appendix 1). Specifically, students were first asked to rank 8 factors, based on prior literature, team discussion, and anecdotal student input, in order of most to least important to their overall wellness (academic performance,[23, 24] physical fitness,[25, 26] family,[27, 28] financial stability,[24] friends,[27, 28] hobbies,[29] alone time,[30] sleep[23, 26, 31]). Then, participants ranked 8 accomplishments, ranging from publishing research and matching into a first-choice residency program to keeping up with friends and maintaining a healthy diet, in order of most to least important to feeling successful as a medical student. Participants also ranked, on a scale from 1 
to 10 , the importance they place on maintaining wellness in medical school. Finally, they were asked to respond in free-text to the questions "What do you do to keep yourself feeling well?" and "What do you do when you are not feeling well?" and subsequently, "What gets in the way of you doing those things?" For the third question, participants selected one or more factors as barriers, based on prior literature, team discussion, and anecdotal student input, including school work, family responsibilities, research, friends, finances, and physical or mental health.

Descriptive statistics were used to summarize participant questionnaire responses. Highly important ranked responses were compared to not important. Grouped responses for feeling well vs not feeling well were also compared. Categorical variables were compared using Chi-square test. P-values $<.05$ were considered significant. We conducted all analyses using SAS Version 9.4 (SAS Institute Inc.).

\section{Qualitative Data Collection}

All 100 students were also invited for an in-person, extended interview, of whom a 20 student subset of the 49 who had consented and completed the first questionnaire agreed to schedule a mutually convenient time with one of the student investigators $(\mathrm{KC})$. Interviews were held between October and December 2018, when first-year students had already been through rigorous early coursework including Human Anatomy, and second year students had begun their Organ-System based instruction. All interviews were conducted by the same student investigator. Participants were informed that the interview would be recorded, transcribed, and analyzed, and that all recorded data would be anonymized. All interviews were held in private rooms at the MCASOM-AZ campus. Participants were able to end the interview at any time with no consequence or loss of reward.

All interviews were guided by the same set of 20 question prompts, followed in order (Fig. 1). Similar to the quantitative survey, Questionnaire 1, these interview prompts were generated by the investigators based on prior literature, team discussion, and anecdotal student input. These prompts were asked with the intention to understand reasons for what we were seeing in the quantitative data and to stimulate conversation around the topic of student wellness, including: personal conceptions of wellness, strategies for maintaining wellness, perceived barriers to wellness, differences in behavior when already feeling well or unwell, the impact of MCASOM-AZ curriculum and resources on wellness, and the portrayal of wellness in medical education. Interviews were not limited in time, though anticipated to last 30-45 minutes.

Qualitative data was analyzed using methods of thematic analysis, which is defined as the systematic process of sorting information based on identified themes.[32-35] Dominant themes, based on multiple participants repeating similar experiences, attitudes, or needs, were identified and a coding strategy was developed. Three investigators $(\mathrm{KV}, \mathrm{JH}$, and $\mathrm{KC}$ ) independently coded all qualitative data, compared categories and refined the parameters of each theme. Any discrepancies in coding to themes prompted review of full interview transcript to better understand meaning through examination of the original context. Aside from the student $\mathrm{KC}$, two of the coding investigators ( $\mathrm{KV}$ and $\mathrm{JH}$ ) have experience in qualitative methods and were brought in to assist with analysis to address reliability and validity of coding and reporting data.[32,34] The two non-student coders ( $\mathrm{KV}$ and $\mathrm{JH})$ were not involved with the 
medical school, study design, sampling approach, or data collection, and not involved in implementing wellness programming in the medical school. Consequently, they may be considered free of biases related to medical school students and wellness curriculum. KC, as a current medical student and as the primary interviewer, brought another perspective to the analysis process. The investigators practiced selfreflection and discussed potential for bias in coding and reporting themes. After these two investigators independently coded qualitative data, predominant theme categories were agreed upon and representative quotes were identified for each theme.

\section{Results}

Quantitative Data Analysis

\section{Participants}

58 of 100 students consented to participate however of those consented, only 49 went on to respond to the baseline questionnaire, 30 to the midterm questionnaire (six month follow-up), and 29 to the final questionnaire (12 month follow-up). Year 1 and year 2 students were almost equally represented (41.4\% and $58.6 \%$ respectively). Gender was almost equally represented throughout (at baseline $51 \%$ female, $49 \%$ male, none non-confirming). Age distribution is shown in Table 1 . There was no difference in age ( $X^{2}$ $(2,49)=3.68, p=.159)$ or gender $\left(X^{2}(1,49)=0.008, p=.928\right)$ between participants who only responded at baseline and those who completed one or both follow-up questionnaires. In addition, the respondents in this study were representative of the student population as a whole based on age and gender. 
Table 1

Demographic breakdown of respondents at baseline, midterm (6-month follow-up), and final (12-month follow-up) time-points.

\begin{tabular}{|c|c|c|c|c|}
\hline Baseline & Midterm & Final & P-value & \\
\hline Age, n (\%) & & & & $0.1588^{1}$ \\
\hline$\leq 23$ & $21(42.9 \%)$ & $6(20.0 \%)$ & $6(20.7 \%)$ & \\
\hline $24-29$ & 22 (44.9\%) & 19 (63.3\%) & $19(65.5 \%)$ & \\
\hline$\geq 30$ & $6(12.2 \%)$ & $5(16.7 \%)$ & $4(13.8 \%)$ & \\
\hline Gender, n (\%) & & & & $0.9280^{1}$ \\
\hline Female & 25 (51.0\%) & $18(60.0 \%)$ & $14(48.3 \%)$ & \\
\hline Male & $24(49.0 \%)$ & $12(40.0 \%)$ & $15(51.7 \%)$ & \\
\hline Non-conforming & $0(0 \%)$ & $0(0 \%)$ & $0(0 \%)$ & \\
\hline Total, $n$ & 49 & 30 & 29 & \\
\hline
\end{tabular}

\section{Conceptions of Wellness and Success}

Results from questionnaire 1 , which ranked factors contributing to their overall wellness are graphically shown in Fig. 2. Family was most important (i.e., ranked 1,2 , or 3$)$ at both baseline $(n=33,67.4 \%)$ and final $(n=23,79.3 \%)$. While $32(65.3 \%)$ students felt academic performance was most important at baseline, this decreased to 14 (48.2\%) at final. Physical fitness increased in importance with 14 (28.6\%) students selecting it as most important at baseline and 14 (44.8\%) at final. Similarly, alone time increased in importance with 5 (10.2\%) selecting it as most important at baseline and 7 (24.9\%) at final. Financial stability was consistently ranked as least important (i.e., ranked 6,7 , or 8 ) to overall wellness with 23 (46.9\%) at baseline and $16(57.2 \%)$ at final.

The two areas that the majority of students felt were important (i.e., ranked 1 or 2 ) to their success as a medical student included doing well on board exams and matching into a first choice residency program. Interestingly, the percentage of students who ranked board exams as important decreased from 37 (75.5\%) at baseline to $17(58.6 \%)$ at final. Matching into a first choice residency program decreased, too, from $29(59.2 \%)$ to $15(51.7 \%)$. Spending time with family which was ranked at third place at baseline became more important to students' success over the year from $24(48.9 \%)$ to $16(55.1 \%)$ at final.

Across all three time points, students who ranked academic performance as important to their overall wellness also selected doing well on board exams and matching into a first-choice residency program as 
important to feeling successful as a medical student. For instance, at baseline, of the 26 (53.1\%) students who indicated academic performance as highly important to their overall wellness (ranked 1 or 2), 21 $(80.8 \%$ vs $39.1 \%)$ selected doing well on board exams $\left(X^{2}(1,49)=18.86, p<.001\right)$ and $17(65.4 \%$ vs $30.4 \%)$ selected matching into a first-choice residency program $\left(X^{2}(1,49)=12.15, p=.015\right)$ as highly important (ranked 1 or 2 ) to feeling successful as a medical student. Conversely, students who rated academic performance as less important to their overall wellness also rated academically-related accomplishments (board exams, first-choice residency program) less highly (not ranked 1 or 2) in whether or not they felt successful as a medical student. At baseline, of the $23(46.9 \%)$ students who indicated academic performance was not as important to their overall wellness (not ranked 1 or 2), 19 (82.6\%) felt publishing research was not highly important (not ranked 1 or 2 ) to their success as a medical student and 19 (82.6\%) indicated the same for becoming involved in extracurricular activities, although these were not statistically significant.

We asked students to indicate how important it was to them to maintain their wellness while in medical school on a scale from 1 (not important) to 10 (most important). The majority of respondents felt this was highly important (9 or 10) at all three time points; 29 (59.2\%) at baseline, 19 (63.4\%) at midterm, and $16(57.2 \%)$ at final. Students who ranked academic performance as highly important (1 or 2 ) to their overall wellness did not feel maintaining their wellness while in medical school was most important (10) as frequently as their peers (10 or $43.5 \%$ of whom ranked it 10$)$. However, $23(88.5 \%)$ still ranked it between 8 and 10 .

\section{Barriers to maintenance of wellness}

Across our three time points across the academic year, we also queried students on whether work, family responsibilities, research, friends, finances, or physical and mental health were a barrier to engaging in activities they did to maintain their own wellness (i.e., their answers to free text questions below), with the option to select all that applied. Almost all respondents felt work was an obstacle with $44(89.8 \%)$ at baseline, $29(96.7 \%)$ at midterm, and $29(100 \%)$ at final. A majority of respondents did not feel that family responsibilities, friends, finances, or their physical or mental health were an impedance in this regard at any of the timepoints. Students were split on research; at midterm, 14 (46.7\%) indicated that research was an impedance while 16 (53.3\%) felt that it was not. MCASOM-AZ requires students to complete one research project over the 4 years and must submit research proposals by the end of year 2 .

\section{Qualitative Data Analysis}

Strategies for Wellness \& Coping

Also on Questionnaire 1, we allowed students to respond in free-text to the questions "What do you do to keep yourself feeling well?" and "What do you do when you are not feeling well?" Students frequently referenced social activities, hobbies (reading, hiking, crafts), spiritual or religious engagements, exercise, diet, sleep, alone time, academic work, and mental health management (counseling and medications) in 
response to both questions. Students frequently mentioned engaging in social activities, such as calling loved ones or spending time with friends to keep themselves feeling well (35 or $68.6 \%$ of responses), but were significantly less likely to mention this when they were not feeling well (19 or $37.3 \%$ of students, $X^{2}$ $(1,51)=10.38, p=.002)$. Conversely, $12(23.5 \%)$ students reported doing a solitary activity to keep themselves feeling well, while $22(43.1 \%)$ students reported doing something alone when they are not feeling well $\left(X^{2}(1,51)=4.37, p=.036\right)$.

Interview Results

A total of 20 (12 female and 8 male) participants agreed to sit down for an in-person interview with a student investigator, which as described in the methods section was semi-structured with a list of 20 prompts generated by the investigators for the purpose of this study. Four of the participants were 1st year students and 16 were 2 nd year students at MCASOM-AZ. All 20 participants who chose to sit down for the interview completed their interview by supplying a response to all 20 prompting questions. The length of each interview ranged between 25 and 40 minutes.

Thematic analysis revealed several agreed upon patterns in student responses, and specific representative quotes are provided in Table 2. Firstly, no one thought that wellness was "over-rated"; students consistently elaborate their personal commitment to, and unique conception of, wellness in ways that elucidate this whole conversation. Most students alluded to the importance of balancing relationships, self-care, and academics in achieving wellness (the word "balance" was mentioned 65 times). Students largely agree that wellness is contextual and inseparable from the broader culture of medical training. Given this, further discussion revealed a theme of subtle skepticism about addressing "wellness" in medical school without addressing a predominant medical culture that contributes to student and physician burnout and insufficiently emphasizes wellness. For instance, one participant cautioned that the medical school could take the unhelpful perspective that "We're gonna push our students really hard. We're gonna have high expectations. This is gonna be a rigorous curriculum. This is gonna stress them out, but it's OK because we have a wellness program... It's treating the symptoms rather than the problem." These medical students strongly discouraged mandatory wellness activities, (e.g., "keeping the exact same system, but also, now there's a yoga class you're supposed to go to, doesn't address the real problem." Or "The only wellness activities I didn't enjoy were when we had them in the classroom, and it felt like they were teaching us how to be well... wellness is something that originates in you and it doesn't need to be so structured."), were more open to student-initiated activities than schoolinitiated programs (e.g., "Whenever I go into these wellness activities and I see it's like a physician or an administrator that's the moderator, there is this implicit bias in the back of my head that you don't know what I'm going through, therefore I'm going to take everything you say with a grain of salt."). Furthermore, a valued wellness activity for one student can be perceived as burdensome to another. Instead, many described the importance of a diverse and inclusive conceptualization of wellness that allows for individual differences, choice, and flexibility. Almost all valued having easily accessible school resources available such as mental health counselors and academic success advisors, some mentioned that they had utilized the services, and several said that while they would first seek support from family and friends 
before going to a counselor. In terms of peer support, almost all mentioned the importance of friends and classmate support, though many found that their classmates also added to stress when they stressed out about school ("The ever-impending doom of like certain classmates talking about it all the time. What they're doing, and just that pressure to compare yourself to other people, which I tried to step away from."). Many acknowledged that the pass/fail system decreased academic stress and made it easier to feel support and camaraderie from their peers. Also, several elaborated on why they are less social when stressed. ("When I'm feeling stressed, that's when I most feel like I need time to myself. Partly because the things that stress me out are often social interactions.") Finally, all interviewees felt comfortable talking with $\mathrm{KC}$, and many mentioned that they enjoyed the opportunity to talk confidentially to a peer and be listened to. These themes align with each other to reveal a broader consensus attitude around the nature of medical student wellness and opportunities to improve it. 
Table 2

Predominant themes identified from sit-down interviews, with representative quotes

\section{Predominant Representative Quote}

Theme

Students place value on wellness and have unique conceptions of wellness
"That could be spiritually, religiously, living a moral life in accordance with your morals. No cognitive dissonance in your life. You're living the life that you feel is right, and you're progressing towards your goals, be they career, family, personal, whatever"

"For me, still a large part of my identity and how I view myself is in my abilities, my academics, and my desire to become a doctor. At the end of the day, I feel like I have multiple views in myself. Centrally, when I introduce myself to someone I say first and foremost I'm a medical student. If I was to focus on all of those other aspects of my wellness that's great, but if I didn't have a chance to actually become a doctor I think that would affect me a lot too."

"For me wellness honestly means that I get to have a balance in my life between the things I prioritize. It means not doing too much of one thing and letting that overtake my life."

"For me, a lot of my wellness stems from my interactions with my family and friends. That's the top thing, to maintain those relationships."

Wellness is viewed as contextual and can't be separated from medical culture
"You take that type of person who clearly doesn't value wellness. That's one party, and there's that subset that still exists to this day where that's what it takes to be a doctor. That's still trickled down into the idea of medicine.... systematic mentality coming down on us from both physicians and these ideas that have been ingrained in students"
There is skepticism towards wellness efforts without addressing predominant medical culture

"It's one of those environments that with certain chronic illnesses and with certain external stressors like being in medical school. It's exacerbated environment"

"Burnout has this connotation that it's a shortcoming on the part of the people experiencing burnout, rather than a shortcoming of the construct of the culture that people who are experiencing burnout are working in"

"We're gonna push our students really hard. We're gonna have high expectations. This is gonna be a rigorous curriculum. This is gonna stress them out, but it's OK because we have a wellness program... It's treating the symptoms rather than the problem."

"The people who value wellness aren't rewarded by the system the way it's set up currently, which is ironic... yes you can incorporate wellness, but until the system rewards it, there's only so far you can take it." 


\section{Predominant Representative Quote \\ Theme}

"In an academic setting, it's [wellness] always an adjunct right? It's like, "We want all our students to perform well academically. Also, here's wellness over here," off to the side. I get frustrated because I feel like it's something that is talked about but not truly addressed."

"Keeping the exact same system, but also, now there's a yoga class you're supposed to go to, doesn't address the real problem."

Mandatory wellness activities are discouraged
"I wonder about the efficacy of an institution almost wagging a finger and saying, "You should." Shoulds are a really good way to have people turn their backs on it.'

"I'Il say that I don't necessarily find mandatory wellness things I need to go to particularly helpful. Going back to my timing and business issues, it sounds like just another thing on the list. I find more intrinsically motivated wellness activities to be more helpful."
Individual differences and preferences for wellness activities should be honored "It's become grouped because that's what's been studied into a specific subset of

"It's the students that defines wellness as physical fitness, socialization and stuff like that that really benefit from the wellness program"

"...there is a little bit of that barrier where I'm not fully convinced that you know where I'm coming from. Perhaps, maybe having student moderators would be a really good way to ease that, as well."

\section{Discussion}

The responses from Questionnaire 1 allow for several, numerically supported claims to be made in favor of factors students associate strongly with their wellness, and factors that are conducive to wellness. These supported claims gain necessary, personal context when taken alongside interview responses. The potential for such cross-analysis was the original motivation for performing this mixed methodology study. Key themes present and mutually supported by both the questionnaire and interview responses can be organized into a series of related discussions.

How is wellness enabled and conceived?

The survey format is suited to gather data on the contributing factors for wellness, how wellness (however conceived by the participant) is enabled. As for these enabling factors, on Questionnaire 1, at all three timepoints Family, Academic Performance, and Friends are consistently the highest ranked in their 
importance to overall wellness. Alone Time and Hobbies are consistently lowly ranked, and Financial Stability remained last in ranking at all three timepoints. These findings are thoroughly reflected in the interview responses, which allowed further exploration into the individual conceptions of wellness that are enabled by these factors. Throughout the interviews, when students are asked to elaborate their idea of wellness, mention of Family and Friends numerous times (70 mentions of family and 91 mentions of friends) and the role of Academic Performance is mentioned in enough detail to warrant separate discussion. Not a single interviewed participant mentioned Financial Stability as a factor contributing to their wellness, and only one person mentioned finances as one of the barriers to wellness. Although this finding may not be generalizable across school, geographic area, and/or year in schooling, the lower relative weight of this factor during preclinical years is supported by prior findings.[20] Of note, while many previous studies outline the various self-care behaviors employed by students,[16] interview data reveals how these behaviors change depending on current stress level, specifically regarding the choice to socialize.

Through conversation, it could be seen that personal definitions of wellness are broad and deep. Nearly all participants included social, physical, emotional wellbeing into their definition, often explicitly citing the "health triangle," but they also identified several factors that weigh particularly heavily for them as individuals but are not often discussed, like travel and romance. There was frequent conversation around spiritual well-being, and multiple students commented on their search for inner peace, quietude, and purpose. The importance of family featured prominently both for coping and academic success, suggesting that disruptions (e.g., death or illness of family member) could disproportionately negatively impact wellness and therefore warrants further study. Although one may expect that the relative influence of Family might wane as immersion in the medical community and career path deepens, this was shown not to be the case according to the survey data, which as mentioned continue to be highly favored during repeat midterm and final assessments and the interviews in which participants explained the value of Family (e.g., "I'll call up my mom, my dad, my grandma, my brothers, sister, just talk to them and connect with them...I feel that helps keep me grounded. They give me perspective."). Finally, the pass/fail system and the awareness of school resources (even if not used) were frequently mentioned as comforting. These factors may partly explain why in our previous study[22] we observed decrease in perceived stress and increase in wellbeing over the course of the year, despite no difference between those who did or did not participate in wellness curriculum.

What is the relationship between wellness and academic performance?

Although unsurprising for this academically-minded study population, Academic Performance is second only to Family as an important factor in overall well-being. One student elaborates, "a large part of my identity and how I view myself is in my abilities, my academics, and my desire to become a doctor." While important for well-being and central to identity, [academic] work is also cited by almost all as an obstacle to wellness. One participant elaborates, "school is often not the stressor, but rather what exacerbates other stressors," and another refers to school as "the exacerbated environment." This begins to explain 
why by the end of the academic year there is a $17.1 \%$ decrease in those who highly rank Academic Performance in their well-being.

Furthermore, results show that students begin to bifurcate into two groups. Students who rank Academic Performance highly in well-being are more likely to choose these extrinsic achievements as important to success in medical school while those who lowly rank Academic Performance are less likely. This bifurcation is reflected in the interview, as some students gauge their success by extrinsic measures ( "a very metric ingrained definition of success"), and others gauge it more holistically ("I have some level of mastery of the material [while] not feeling the class taking over my life"). Several students mentioned that being top was not worth forfeiting wellness ("Even if I was getting the top score on an exam, if I was sacrificing sleep or friendships to do that, I wouldn't feel like that was a success. I would feel like that was not worth the price."). Those who ranked Academic Performance highly for well-being were also less likely to report on Questionnaire 1 that maintaining wellness in medical school is important, as if wellness is contained within academic performance and does not require additional attention. To our knowledge, this dichotomy has not been shown in previous studies. Given that we found a decrease over time in the percentage who ranked academic performance as key to wellness, it may be that students begin to shift priorities toward internal rewards versus external rewards. Better understanding these associations is an area ripe for more research.

How does the atmosphere of medical education relate?

Seeing as academic work is a major factor of overall student wellness, it follows that student wellness is contextualized within the academic environment. Even early in their medical education, participants have clear opinions towards the atmosphere of medical education, commonly labeled "the system," and regard it as a hindrance to wellness. Students comment that wellness is treated separately from the rest of formal medical education, saying "In an academic setting, it's [wellness] always an adjunct right?" Student wellness is perceived as not only separate, but also inadequately tended to, as this same student continues, "I feel like it's something that is talked about but not truly addressed." Students frequently commented on wellness curricula as insufficient and misguided with four students referring to them as a "band-aid."

When prompted further about the origins of this institutional attitude towards student wellness, students share numerous insights. They articulate the belief that this attitude is inherited, saying "it's a systematic mentality coming down on us from physicians," and that it is perpetuated by an incentive structure whereby "the people who value wellness aren't rewarded by the system." One student explains this as the result of "a lot of inefficiencies in our system right now." Another focuses the issue on how "Wellness is often portrayed in only its negative form, burnout, which also has this connotation that it's a shortcoming on the part of the people experiencing burnout rather than a shortcoming of the construct." Altogether, a clear theme of skepticism emerges towards wellness efforts that fail to address the predominant medical culture. This sentiment runs alongside a longstanding and well-known aspect of medical training commonly referred to as the "hidden curriculum," whereby trainees are placed in a predominant medical 
culture that teaches behaviors in the clinical workplace that are different or antithetical to what is explicitly taught.[10, 36]

What curricular improvements follow from this discussion?

Just as wellness was found to be diversely conceptualized through our in-depth interviews, a wellness curriculum should not be narrowly biased towards conventional and stereotypic conceptions. Students describe this bias for "people who prefer exercise and mindfulness" and for "activities more leisurely in nature," and as a narrow "subset of ideas and activities like yoga." Instead, activities that are cerebral, or otherwise less stereotypically associated with wellness may be more inclusive and well-received. As one student summarized, "One thing that I think is not emphasized enough in wellness is self-efficacy and locus of control."

Given the close and conflicted relationship between wellness and academics as mutually elucidated through the survey and interview data, it follows that wellness curricula ought to be conceived alongside academic curricula and that academic work (cited universally as the obstacle to wellness activities) be made more manageable[18]. One student expresses "Maybe the answer is not necessarily to have more extra things, but maybe to structure the academic part of school in such a way that it's not as taxing." This is an echo of a prior meta-analysis study showing marked improvement in well-being with a pass/fail grading system,[12] as well as another Australian study which found favorable response to approved "well-being day" absences.[19] In addition to making curricula more manageable, faculty may also integrate wellness in how they present existing coursework, thereby demonstrating wellness as an integral physician competency and building trust between staff and students, which this same Australian study found lacking.[19]

Furthermore, the emphasis placed on family and independent strategies for maintaining wellness advise a student-directed approach to wellness programming.[15] Interviews reveal adamant opposition to mandatory wellness activities, enough to warrant identification as a core theme, with one student saying "Should's are a really good way to have people turn their backs." Both this study and our previous report suggests that student-driven, as opposed to faculty-driven, are more likely to enjoy greater participation and increased student wellness.[22] These findings mirror prior literature, which urges caution in further "institutionalizing" wellness initiatives rather than to have them arise organically from the student body itself.[14] A pronounced trend has been noted in the climate of current medical education where students are choosing to learn content individually and through mixed resources as opposed to traditional scheduled classroom didactics.[37] With these prevailing trends in student attitude, optimal engagement can be expected when wellness initiatives are similarly student-driven, as previously found in one prior study in Singapore.[15] In line with this finding, both in their interview and free-text survey response, many students express support for student-initiated wellness grants at MCASOM-AZ that fund activities proposed by students themselves or for programming that is student-led. To the extent that students find some of the interactions stressful with other students, student-led programming might include strategies for discussing academic worries with each other that are geared toward supportive listening and 
normalizing common worries. Lastly, and consistent with the main direction of this study to focus on individual conceptions of wellness, many students identified a need for more reflection on the attitudes surrounding their personal wellness and see the value of such reflection. This research interview was noted by many participants as a welcomed occasion for such reflection, suggesting a potential role for peer coaching as a strategy to foster reflection and peer support. On the basis of these and other findings, our school eliminated mandatory lectures and adopted additional peer support programming and student-led wellness curricula, which indeed has had high participation rates. We are currently evaluating the impact of the new programming.

The findings of this study are limited by several features. Firstly, results are limited by small sample sizes for questionnaire responses at all three timepoints, as well as many dropouts at the midterm and final timepoints. This may introduce the bias that students who are feeling particularly unwell are excluded from later responses. Furthermore, study findings reflect only the experience of students in the preclinical years 1 and 2, where there is not yet the additional stressor and rewarding experience of hands-on patient care. Additionally, although having a student interviewer may have allowed for greater trust from participants, it could have introduced an element of bias, whereby students may have felt the pressure to echo sentiments that they may have believed the interviewer or the greater student body may have held.

\section{Conclusions}

Mixed methods qualitative research can provide important insights to understand and explain the quantitative and categorical data. As predicted, medical students themselves hold a wealth of insight into what wellness means for them and how it enables their continued personal and professional success. These insights proved as diverse as the students themselves, while also revealing certain recurrent themes. A central theme was the conflicted relationship between wellness and academic performance, wherein academics is critical to student identity and their definition of wellness while also the largest barrier. Students diverged and fluctuated in how closely they perceived this relationship between academic performance and wellness. Another resounding theme was the emphasis placed on family and independent activities, especially when the intent is to recover well-being instead of maintaining it. Curricular improvements follow directly from these themes and student insight into the current atmosphere of medical education. Medical schools may find similar attitudes amongst their students and may thereby benefit from these curricular improvements, especially if implemented within a broader, organized effort as prescribed in prior literature[1] and at other medical schools. $[13,18]$

\section{Declarations}

Ethics approval and consent to participate The Mayo Clinic Institutional Review Board and the Mayo Clinic College of Medicine and Science Education Research Committee approved this study (protocol number 18-005605). Informed consent was obtained from all individual participants in the study. Approval from the Mayo Clinic Institutional Review Board additionally ensures that guidelines regarding research involving human subjects have been followed in accordance with the Declarations of Helsinki. 
Consent for publication: Not applicable

Availability of data and materials: The datasets generated and/or analyzed during the current study are not publicly available due to the nature of qualitative data as audio files of specific interviewees who have not consented for their individual voices to be heard by parties aside from direct investigators. Quantitative data from survey results are outlined clearly in the manuscript but records from survey software used to gather data (REDCap) are available from the corresponding author on reasonable request.

Competing Interests: The authors declare that they have no competing interests.

Funding: Benefactor gift to Dr. Stonnington and Mayo Clinic for support of Wellbeing activities

Authors' Contributions: $\mathrm{KC}$ had a major role in study design, collection of data, analysis of qualitative data and writing the manuscript. VE had a major role in study design, collection of data, and providing edits to all drafts of the manuscript. MG performed all statistical analyses and contributed to the writing of the manuscript. KV supervised and performed the qualitative data analysis and contributed to the writing of the manuscript. JH performed the qualitative data analysis. CS supervised the study design and data collection and provided substantial edits to all drafts of the manuscript. All authors read and approved the final manuscript.

Acknowledgments: Not applicable

Author's information: Not applicable

\section{References}

1. Shanafelt T, Trockel M, Ripp J, Murphy ML, Sandborg C, Bohman B: Building a Program on WellBeing: Key Design Considerations to Meet the Unique Needs of Each Organization. Academic Medicine 2019, 94(2):156-161.

2. Shanafelt TD, Hasan O, Dyrbye LN, Sinsky C, Satele D, Sloan J, West CP: Changes in Burnout and Satisfaction With Work-Life Balance in Physicians and the General US Working Population Between 2011 and 2014. Mayo Clinic Proceedings 2015, 90(12):1600-1613.

3. Ludwig AB, Burton W, Weingarten J, Milan F, Myers DC, Kligler B: Depression and stress amongst undergraduate medical students. BMC Med Educ 2015, 15:141.

4. Dyrbye LN, Satele D, Sloan J, Shanafelt TD: Utility of a Brief Screening Tool to Identify Physicians in Distress. Journal of General Internal Medicine 2013, 28(3):421-427.

5. Dyrbye LN, Satele D, Sloan J, Shanafelt TD: Ability of the physician well-being index to identify residents in distress. Journal of graduate medical education 2014, 6(1):78-84.

6. Dyrbye LN, Massie F, Eacker A, et al.: Relationship between burnout and professional conduct and attitudes among us medical students. JAMA 2010, 304(11):1173-1180. 
7. Dyrbye LN, Thomas MR, Shanafelt TD: Systematic Review of Depression, Anxiety, and Other Indicators of Psychological Distress Among U.S. and Canadian Medical Students. Academic Medicine 2006, 81(4):354-373.

8. Seritan AL, Rai G, Servis M, Pomeroy C: The office of student wellness: innovating to improve student mental health. Acad Psychiatry 2015, 39(1):80-84.

9. Dyrbye LN, Thomas MR, Massie F, Power DV, Eacker A, Harper W, Durning S, Moutier C, Szydlo DW, Novotny PJ et al: Burnout and suicidal ideation among U.S. medical students. Ann Intern Med 2008, 149(5):334-341.

10. White $C B$, Kumagai AK, Ross PT, Fantone JC: A qualitative exploration of how the conflict between the formal and informal curriculum influences student values and behaviors. Acad Med 2009, 84(5):597-603.

11. Agarwal G, Lake M: Personal Transition to the Profession: A Novel Longitudinal Professional Development and Wellness Medical Student Curriculum. Academic Psychiatry 2016, 40(1):105-108.

12. Wasson LT, Cusmano A, Meli L, Louh I, Falzon L, Hampsey M, Young G, Shaffer J, Davidson KW: Association Between Learning Environment Interventions and Medical Student Well-being: A Systematic Review. JAMA 2016, 316(21):2237-2252.

13. Drolet BCMD, Rodgers SMD: A Comprehensive Medical Student Wellness Program-Design and Implementation at Vanderbilt School of Medicine. Academic Medicine 2010, 85(1):103-110.

14. Cole A: Is the Institutionalization of Wellness the Answer to Trainee Burnout? Am J Psychiatry Resid J 2018, 13(7):7-8.

15. Farquhar J, Kamei R, Vidyarthi A: Strategies for enhancing medical student resilience: student and faculty member perspectives. Int J Med Educ 2018, 9:1-6.

16. Ayala EE, Omorodion AM, Nmecha D, Winseman JS, Mason HRC: What Do Medical Students Do for Self-Care? A Student-Centered Approach to Well-Being. Teaching and Learning in Medicine 2017, 29(3):237-246.

17. Kligler B, Linde B, Katz NT: Becoming a doctor: a qualitative evaluation of challenges and opportunities in medical student wellness during the third year. Acad Med 2013, 88(4):535-540.

18. Slavin SJ, Schindler DL, Chibnall JT: Medical student mental health 3.0: improving student wellness through curricular changes. Academic Medicine 2014, 89(4):573-577.

19. Byrnes C, Ganapathy VA, Lam M, Mogensen L, Hu W: Medical student perceptions of curricular influences on their wellbeing: a qualitative study. BMC Med Educ 2020, 20(1):288.

20. Hill MR, Goicochea S, Merlo LJ: In their own words: stressors facing medical students in the millennial generation. Med Educ Online 2018, 23(1):1530558.

21. Jenkins TM, Kim J, Hu C, Hickernell JC, Watanaskul S, Yoon JD: Stressing the journey: using life stories to study medical student wellbeing. Adv Health Sci Educ Theory Pract 2018, 23(4):767-782.

22. Edmonds V, Chatterjee K, Girardo M, Butterfield R, Stonnington CM: Evaluation of a Novel Wellness Curriculum on Medical Student Wellbeing and Engagement Demonstrates a Need for Student-Driven 
Wellness Programming. Teaching and Learning in Medicine 2022, In press.

23. Lapinski J, Yost M, Sexton P, LaBaere RJ, 2nd: Factors Modifying Burnout in Osteopathic Medical Students. Acad Psychiatry 2016, 40(1):55-62.

24. Matheson KM, Barrett T, Landine J, McLuckie A, Soh NL, Walter G: Experiences of Psychological Distress and Sources of Stress and Support During Medical Training: a Survey of Medical Students. Acad Psychiatry 2016, 40(1):63-68.

25. Cecil J, McHale C, Hart J, Laidlaw A: Behaviour and burnout in medical students. Med Educ Online 2014, 19:25209.

26. Wolf MR, Rosenstock JB: Inadequate Sleep and Exercise Associated with Burnout and Depression Among Medical Students. Acad Psychiatry 2017, 41(2):174-179.

27. Bore M, Kelly B, Nair B: Potential predictors of psychological distress and well-being in medical students: a cross-sectional pilot study. Adv Med Educ Pract 2016, 7:125-135.

28. Thompson G, McBride RB, Hosford CC, Halaas G: Resilience Among Medical Students: The Role of Coping Style and Social Support. Teach Learn Med 2016, 28(2):174-182.

29. Mavor KI, McNeill KG, Anderson K, Kerr A, O'Reilly E, Platow MJ: Beyond prevalence to process: the role of self and identity in medical student well-being. Med Educ 2014, 48(4):351-360.

30. Kalet A, Krackov S, Rey M: Mentoring for a new era. Acad Med 2002, 77(11):1171-1172.

31. Mazurkiewicz R, Korenstein D, Fallar R, Ripp J: The prevalence and correlations of medical student burnout in the pre-clinical years: a cross-sectional study. Psychol Health Med 2012, 17(2):188-195.

32. Hsieh HF, Shannon SE: Three approaches to qualitative content analysis. Qual Health Res 2005, 15(9):1277-1288.

33. Kirk J, Miller ML: Reliability and validity in qualitative research vol. 1; 1986.

34. Neuendorf K: The Content Analysis Guidebook, 2nd Edition edn. Thousand Oaks, CA: Sage Publications, Inc.; 2017.

35. Patton MQ: Qualitative evaluation and research methods Thousand Oaks, CA: Sage Publications, Inc.; 2014.

36. Lempp H, Seale $\mathrm{C}$ : The hidden curriculum in undergraduate medical education: qualitative study of medical students' perceptions of teaching. BMJ 2004, 329(7469):770-773.

37. Wu JH, Gruppuso PA, Adashi EY: The Self-directed Medical Student Curriculum. JAMA 2021, 326(20):2005-2006.

\section{Figures}

\section{Figure 1}

Guide for Peer-to-Peer Interview 
List of question prompts used to guide sit-down wellness interview

\section{Figure 2}

Factors for Wellness Ranked

Percentage of students that ranked a given factor either 1,2 , or 3 across each timepoint

\section{Supplementary Files}

This is a list of supplementary files associated with this preprint. Click to download.

- Wellnessadditionalfile1.pdf 